\title{
A Criterion for Compatibility of Conformal and Projective Structures
}

\author{
Vladimir S. Matveev ${ }^{1}$, Andrzej Trautman ${ }^{2}$ \\ 1 Fakultät für Mathematik und Informatik, Mathematics Institute, Friedrich-Schiller-Universität Jena, \\ 07737 Jena, Germany. E-mail: vladimir.matveev@uni-jena.de \\ 2 Institute of Theoretical Physics, Hoża 69, 00681 Warsaw, Poland. E-mail: andrzej.trautman@fuw.edu.pl
}

Received: 10 February 2013 / Accepted: 27 March 2013

Published online: 23 November 2013 - (C) The Author(s) 2013. This article is published with open access at Springerlink.com

\begin{abstract}
In a space-time $M$, a conformal structure is defined by the distribution of light-cones. Geodesics are traced by freely falling particles, and the collection of all unparameterized geodesics determines the projective structure of $M$. The article contains a formulation of the necessary and sufficient conditions for these structures to be compatible, i.e. to come from a metric tensor which is then unique up to a constant factor. The theorem applies to all dimensions and signatures.
\end{abstract}

\section{Introduction and Remarks on the History of the Problem}

Hermann Weyl, in his early papers on 'infinitesimal geometry' [1,2], described the two structures that underlie the geometry and physics of the four-dimensional space-time. The propagation of light determines light cones; the collection of all such cones gives a conformal structure $\mathscr{C}$ of Lorentzian signature. Weyl pointed out that gravitation is described by a linear connection: particles, freely falling in a gravitational field, trace unparameterised geodesics that define symmetric linear connections, but only up to 'projective transformations'. The collection of all such projectively related connections is a projective structure $\mathscr{P}$ on a manifold. (Precise definitions are given in the next section.) A Riemannian metric of Lorentzian signature uniquely determines both these structures; Weyl has shown that two metrics $g$ and $g^{\prime}$ give the same two structures (conformal and projective) if and only if $g^{\prime}=$ const $\cdot g$; see Satz 1 in [2]. Weyl did not, however, consider the problem of whether a given pair of conformal and projective structures come from one metric tensor. Simple examples show that, in general, they do not.

The problem raised by Weyl has attracted, over the years, a considerable interest among physicists. Ehlers, Pirani and Schild wrote, on this subject, an influential paper that was recently reprinted as a 'Golden Oldie' [3]. These authors argue in favour of founding the geometry of space-time on its conformal and projective structures rather 
than on the 'chronometric' approach of Synge $[4,5]$. They formulate a necessary condition that the pair $(\mathscr{C}, \mathscr{P})$ must satisfy in order to result from one metric tensor. Namely, according to this EPS condition, as it will be called here, the null geodesics of the conformal geometry should be also geodesics, or autoparallels, as defined by the projective structure. Ehlers, Pirani and Schild formulate further conditions that the structures $\mathscr{C}$ and $\mathscr{P}$ should satisfy so as to come from a unique, up to a constant factor, metric tensor. However, they do not give sufficient conditions for this to be the case. More comments on that paper and further references can be found in [6].

In this paper we present a theorem giving the necessary and sufficient conditions for compatibility of conformal and projective structures. The theorem is algorithmic in the sense that, to determine compatibility of $\mathscr{C}$ and $\mathscr{P}$, it suffices to compute a few simple expressions formed from the components of $g \in \mathscr{C}$ and $\Gamma \in \mathscr{P}$. Our result is also effective: if these two structures are compatible, then a simple integration suffices to find the corresponding metric tensor.

\section{Definitions and the Theorem}

We consider smooth-of class $C^{\infty}$-manifolds and maps. All geometric objects on an $n$-dimensional manifold are referred to local coordinates $\left(x^{i}\right), i=1, \ldots, n$.

A conformal structure on a manifold $M$ is an equivalence class $\mathscr{C}$ of metric tensors $g$ with respect to the following equivalence relation:

$$
g \sim g^{\prime} \Longleftrightarrow \text { there is a function } \varphi \text { on } M \text { such that } g^{\prime}=g \exp 2 \varphi .
$$

If $g \in \mathscr{C}$, then $\mathscr{C}$ can be denoted by $[g]$. No assumption is made on the signature of the metric tensors; they can be properly Riemannian.

Two symmetric linear connections $\Gamma=\left(\Gamma_{j k}^{i}\right)$ and $\Gamma^{\prime}=\left(\Gamma_{j k}^{\prime i}\right)$ are said to be projectively equivalent if their geodesics differ only by parametrisation. Projective equivalence is clearly an equivalence relation on the set of all symmetric linear connections on $M$. An equivalence class $\mathscr{P}$ with respect to this relation is called a projective structure; it is denoted by $[\Gamma]$ if it contains $\Gamma$.

Projective equivalence can be formulated as the condition

$$
\Gamma \sim \Gamma^{\prime} \in \mathscr{P} \Longleftrightarrow \text { there is a } 1 \text {-form } \psi \text { so that } \Gamma_{j k}^{i}=\Gamma_{j k}^{i}+\delta_{j}^{i} \psi_{k}+\delta_{k}^{i} \psi_{j}
$$

In this form it appears in [2], but the essence of this result was given already by Tullio Levi-Civita in his very first publication [7], written at the age of 23 .

Tracy Thomas [8] observed that, given two symmetric linear connections, it is easy to check whether they are projectively equivalent by computing the traceless quantity $\Pi(\Gamma)$, which is nowadays called the Thomas symbol,

$$
\Pi_{j k}^{i}(\Gamma)=\Gamma_{j k}^{i}-\frac{1}{n+1} \delta_{j}^{i} \Gamma_{p k}^{p}-\frac{1}{n+1} \delta_{k}^{i} \Gamma_{p j}^{p}, \quad n=\operatorname{dim} M .
$$

Namely, two symmetric linear connections are projectively equivalent, if and only if, their Thomas symbols coincide,

$$
\Pi(\Gamma)=\Pi\left(\Gamma^{\prime}\right) \Longleftrightarrow \Gamma \text { and } \Gamma^{\prime} \text { are projectively equivalent. }
$$

Let $\digamma(g)$ be the Levi-Civita connection defined by $g$. In local coordinates,

$$
\digamma_{j k}^{i}(g)=\frac{1}{2} g^{i p}\left(\partial_{k} g_{p j}+\partial_{j} g_{p k}-\partial_{p} g_{j k}\right),
$$


so that

$$
\digamma_{j k}^{i}(g \exp 2 \varphi)=\digamma_{j k}^{i}(g)+\delta_{j}^{i} \partial_{k} \varphi+\delta_{k}^{i} \partial_{j} \varphi-g^{i p} g_{j k} \partial_{p} \varphi,
$$

where $\partial_{k} \varphi=\partial \varphi / \partial x^{k}$, etc. If $u=\left(u^{i}\right)$ is a null vector, $g_{i j} u^{i} u^{j}=0$, then

$$
\digamma_{j k}^{i}(g \exp 2 \varphi) u^{j} u^{k}-\digamma_{j k}^{i}(g) u^{j} u^{k} \| u^{i},
$$

so that (unparameterised) null geodesics are well defined in conformal geometry in the sense the null geodesics of $g$ are reparametrised null geodesics of $g \exp (2 \varphi)$.

Definition. The conformal and projective structures $\mathscr{C}$ and $\mathscr{P}$ are said to be compatible if there is $g \in \mathscr{C}$ such that $\digamma(g) \in \mathscr{P}$.

Given $g \in \mathscr{C}$ and $\Gamma \in \mathscr{P}$, from (1) one obtains

$$
\mathscr{C} \text { and } \mathscr{P} \text { are compatible } \Longleftrightarrow \exists \varphi \text { such that } \Pi(\digamma(g \exp 2 \varphi))=\Pi(\Gamma) .
$$

Since the difference of two connection coefficients is a tensor, so is

$$
T_{j k}^{i} \stackrel{\text { def }}{=} \Pi_{j k}^{i}(\digamma(g)-\Gamma) .
$$

The components of this tensor depend on the components of the metric tensor and their first derivatives and on the components of the linear connection. Substituting (2) into (3), one infers that compatibility of $\mathscr{C}$ and $\mathscr{P}$ is equivalent to the existence of $\varphi$ such that

$$
T_{j k}^{i}-g_{j k} g^{i p} \partial_{p} \varphi+\frac{1}{n+1} \delta_{j}^{i} \partial_{k} \varphi+\frac{1}{n+1} \delta_{k}^{i} \partial_{j} \varphi=0 .
$$

Let

$$
T^{i}=\frac{n+1}{(n+2)(n-1)} g^{j k} T_{j k}^{i} \quad \text { and } \quad T_{i}=g_{i j} T^{j} .
$$

By contraction of (4) with $g^{j k}$ one obtains

$$
\partial_{i} \varphi=T_{i} .
$$

Substituting $\partial_{i} \varphi$ determined by (6) and (5) into (4), one obtains the following condition on $g$ and $\Gamma$ :

$$
T_{j k}^{i}-g_{j k} T^{i}+\frac{1}{n+1} \delta_{j}^{i} T_{k}+\frac{1}{n+1} \delta_{k}^{i} T_{j}=0 .
$$

Since the second partial derivatives of $\varphi$ commute, from (6) one obtains

$$
\partial_{j} T_{i}-\partial_{i} T_{j}=0 .
$$

Theorem. The conditions (7) and (8) are necessary and sufficient for local compatibility of the conformal and projective structures, defined on $M$ by $g$ and $\Gamma$, respectively. If, moreover, the first cohomology group of $M$ vanishes, then there is global compatibility.

Proof. The conditions are necessary because they were derived under the assumption of compatibility. Condition (8) implies the existence of a local-in a neighbourhood of every point-solution $\varphi$ of (6). Replacing now $T_{i}$ in (7) by $\partial_{i} \varphi$ one obtains that condition (4) holds. If the first cohomology group of $M$ vanishes, then the closed form $T_{i} \mathrm{~d} x^{i}$ is exact and thus $\varphi$ is defined all over $M$. 


\section{A Simple Application}

Using the theorem one can confirm the existence of pairs $(\mathscr{C}, \mathscr{P})$ that are incompatible even though the EPS condition holds. Indeed, let $g$ be a Lorentzian metric on an $n$ dimensional manifold $M, n \geqslant 3$, and $\mathscr{C}=[g]$. Given a vector field $\left(S^{i}\right)$ on $M$, one considers the projective structure $\mathscr{P}=[\Gamma]$ such that

$$
\Gamma_{j k}^{i}=\digamma_{j k}^{i}(g)-S^{i} g_{j k}
$$

If $u^{i}$ is a null vector, $g_{i j} u^{i} u^{j}=0$, then $\left(\Gamma_{j k}^{i}-\digamma^{i}{ }_{j k}(g)\right) u^{j} u^{k}=0$ so that a null geodesic with respect to $\mathscr{C}$ is also a geodesic with respect to $\mathscr{P}$ and the EPS condition is satisfied.

Computing now $T_{j k}^{i}$ for $\Gamma$ given by (9), one obtains $T^{i}=S^{i}$ and that the algebraic condition (7) is satisfied. Therefore, the pair $(\mathscr{C}, \mathscr{P})$ now under consideration is compatible if, and only if, the form $g_{i j} S^{j} \mathrm{~d} x^{i}$ is closed. In other words, to obtain a manifold with a pair $(\mathscr{C}, \mathscr{P})$ that satisfies the EPS condition but is incompatible, it suffices to take $\mathscr{C}$ containing a Lorentzian metric $g$ and $\mathscr{P}=[\Gamma]$ given by (9), where $S^{i}$ is vector field with a non-integrable distribution of subspaces orthogonal to it.

\section{Concluding Remarks}

The result presented in this paper, though technically very simple, completes a line of research initiated by Weyl and continued by physicists. Many mathematical objects consist of two - or more - structures on one set, connected by a notion of compatibility. Conformal and projective structures on manifolds have a clear origin in physics and, for this reason, their compatibility has attracted the interest of theoreticians.

The left-hand sides of (7) and (8) are tensors of the type given by the position of their indices. Moreover, they are determined by $\mathscr{P}$ and $\mathscr{C}$, but do not depend on the representatives of these equivalence classes. In fact, if $g$ is replaced by $g \exp 2 \varphi$, then the tensor $T_{j k}^{i}$ is replaced by

$$
T_{j k}^{i}-g_{j k} g^{i p} \partial_{p} \varphi+\frac{1}{n+1} \delta_{j}^{i} \partial_{k} \varphi+\frac{1}{n+1} \delta_{k}^{i} \partial_{j} \varphi
$$

and $T_{i}$ is replaced by $T_{i}-\partial_{i} \varphi$, but the left-hand sides of (7) and (8) do not change. One can consider these tensors as a measure of noncompatibility of the projective and conformal structures. These tensors could be used in the construction and study of those nonmetric relativistic theories of space-time that use the conformal and projective structures as the principal building blocks.

It is worth noting here that a conformal structure can be easily reconstructed from the knowledge of the distribution of light cones. Indeed, if $v \in T_{x} M$ is a null vector, then $g_{i j} v^{i} v^{j}=0$ is a linear equation for the components of the metric tensor and, by taking $n(n+1) / 2-1$ generic null vectors at a point, one obtains a system of linear equations whose solution space is one dimensional and gives the conformal structure at that point. The somewhat subtler procedure of reconstructing a symmetric linear connection from the set of all unparametrized geodesics can also be reduced to solving a system of linear equations; see [9, §2.1] for details.

Closely related to the question considered here-but much more difficult — is the Roger Liouvelle problem initiated in [10]: given a system of differential equations

$$
\ddot{x}^{i}=\Lambda^{i}(x, \dot{x}), \quad i=1, \ldots, n, \quad \dot{x}^{i}=\mathrm{d} x^{i} / \mathrm{d} t,
$$


to find the conditions on the functions $\Lambda$ so that the solutions of (10) represent geodesics of a Levi-Civita connection. Recently, the problem has been solved, in two dimensions, by Robert Bryant, Maciej Dunajski and Michael Eastwood [11].

It is also worth noting that there exist projective structures $\mathscr{P}$ such that there is no metric $g$ satisfying $\digamma(g) \in \mathscr{P}$. Indeed, by the results of [12], the existence of such a metric is equivalent to the existence of covariant constant sections of a non-trivial vector bundle with connection. For almost all projective structures such parallel sections do not exist. From general theory there follows the existence of complete systems of differential invariants, i.e. invariant algebraic expressions in the components of $\Gamma$ and its derivatives that determine whether there exists a metric corresponding to the projective structure $[\Gamma]$, see e.g. [13].

Acknowledgements. The authors, who have never met in person, thank Paweł Nurowski for the initiative and encouragement to write this paper.

Open Access This article is distributed under the terms of the Creative Commons Attribution License which permits any use, distribution, and reproduction in any medium, provided the original author(s) and the source are credited.

\section{References}

1. Weyl, H.: Reine Infinitesimalgeometrie. Math. Z. 2, 384-411 (1918)

2. Weyl, H.: Zur Infinitesimalgeometrie: Einordnung der projektiven und der konformen Auffasung. Nachr. Gesellschaft Wiss. Göttingen, Math.-Phys. Kl., 1921, pp. 99-112

3. Ehlers, J., Pirani, F.A.E., Schild, A.: The geometry of free fall and light propagation. In: L. O'Raifeartaigh, ed. General Relativity: Papers in honour of J. L. Synge. Oxford: Clarendon Press, 1972, pp. 63-84, reprinted in Gen. Rel. Grav. 44, 2012, pp. 1587-1609

4. Synge, J.L.: Relativity: The General Theory. Amsterdam: North-Holland, 1960

5. Synge, J.L.: Relativity based on chronometry. In: Recent Developments in General Relativity. Oxford and Warszawa: Pergamon Press and PWN, 1962, pp. 441-448

6. Trautman, A.: Editorial note to: J. Ehlers, F. A. E. Pirani, A. Schild: The geometry of free fall and light propagation. Gen. Rel. Grav. 44, 1581-1586 (2012)

7. Levi-Civita, T.: Sulle trasformazioni delle equazioni dinamiche. Ann. di Mat. serie $2^{a} \mathbf{2 4}, 255-300$ (1896), English transl. by S. Benenti in Reg. Chaotic Dyn. 14, 580-614 (2009)

8. Thomas, T.Y.: On the projective and equi-projective geometries of paths. Proc. Natl. Acad. Sci. USA 11, 199-203 (1925)

9. Matveev, V.: Geodesically equivalent metrics in general relativity. J. Geom. Phys. 62, 675-691 (2012)

10. Liouville, R.: Sur une classe d'équations différentielles, parmi lesquelles, en particulier, toutes celles des lignes géodésiques se trouvent comprises. Comptes Rend. Hebdomadaires des Séances de l'Acad. des Sci. 105, 1062-1064 (1887)

11. Bryant, R.L., Dunajski, M., Eastwood, M.: Metrisability of two-dimensional projective structures. J. Differ. Geom. 83, 465-499 (2009)

12. Eastwood, M., Matveev, V.: Metric connections in projective differential geometry. In: Symmetries and Overdetermined Systems of Partial Differential Equations, Volume 144 of IMA Vol. Math. Appl. New York: Springer, 2008, pp. 339-350

13. Nurowski, P.: Projective versus metric structures. J. Geom. Phys. 62, 657-674 (2012)

Communicated by P. T. Chruściel 